\section{Talk of echinoderms at Tampa Bay}

\section{David Nichols}

Echinoderm Studies I.

Edited by Michel Jangoux and John M. Lawrence.

A.A. Balkema: 1983. Pp.200. Dfl. 70, £15.90.

BY ANY standards, the spiny-skinned echinoderms are successful marine organisms. Huge numbers of starfish are found in the rocky intertidal regions worldwide, sea-urchins are principal browsers in the shallow sublittoral reefs that border the world's oceans and, in deep water, sea-cucumbers can occur in such numbers that in places their biomass is said sometimes to exceed that of all other animals put together.

As most of them possess a fairly wellassociated skeleton, they have been readily fossilized throughout Phanerozoic time and so the phyletic infrastructure of the whole group, with its five living classes and about 15 extinct ones, has received considerable attention, particularly given the plausible arguments put forward that echinoderms are the springboard for the evolutionary jump from invertebrates to chordates. Can such factors explain their fascination? Whether one regards them as unique in the animal world, enigmatic in their complexity, or simply convenient eggfactories for developmental and cell biologists, they have attracted increasing attention over the last 20 years.

Echinoderm specialists first met together at the 14th International Zoological Congress in Washington in 1963, and the resulting volume (Boolootian, R.A. (ed.), Physiology of Echinodermata, Wiley Interscience, 1966) was a monumental review of the group, system by system. At the most recent meeting at Tampa Bay, a series of plenary lectures was introduced, and Echinoderm Studies I contains expanded versions of these reviews. It is the first of an occasional series that will attempt to bring the Boolootian volume up-to-date, but narrowing the fields covered, and giving the authors more scope for speculation.

Three of the articles stress the convenience of echinoderms as subjects for the study of widely different phenomena in the marine environment. In the first place, they have unusually good regenerative powers. Early oyster-fishermen brought up starfish predators from the beds, tore them in half and flung them back into the sea; but it was not long before they realized that there was a good chance that both halves would regenerate, doubling the menace. In her article on phenotypic variation, Marcus reflects on this ability to produce genetically identical progeny: experimental work in this field often requires cloned individuals and echinoderms represent potential subjects for such work.

Secondly, reflecting a younger field of study, the article by Craig on genomic variability in echinoids also concludes that this group, with its extensive fossil record indicating relationships and the ease by which radiolabelling can be performed, are particularly useful as subjects for mapping genome patterns.

Thirdly, an excellent review by Ebert points to the convenience of using echinoids in studying recruitment in marine communities, especially now that techniques exist for culturing the young stages and rearing them beyond hitherto impeditive metamorphosis.

The physiology and functional morphology of echinoderm respiratory systems have received relatively little attention and therefore Shick's wide-ranging review is most useful. He points particularly to the so-called 'internal problem': echinoderms have a large anaerobic component, perhaps accounting for the imbalance of certain energy equations in the massive internal tissues, especially when the gonads are ripe. Shick's treatment is an excellent example of the coherent blending of morphological and physiological aspects.

Echinoderms are not renowned for their learning ability, yet there have been reports of simple habituation which Valentincix, in his contribution on innate and learned responses, suggests should be treated with caution. However, his own work on conditioning of a 'ready to escape' response in starfish is usefully covered.

One of the unique features of echinoderms is the array of semiindependent effector organs with which the outer body is endowed. Down among the spines are the defensive and cleansing pedicellariae, which have their own reflex activity, yet are linked to the 'central' nervous system for concerted action when required. Campbell gives a splendid review here, placing his own work in historical perspective, and showing the sophistication in miniature of these fascinating structures.

The echinoderms, distinct from all other animal groups in so many features but with a good fossil record, have naturally attracted the attention of palaeontologists who seek to understand their evolution. Sprinkle's article reviews some of the controversies that have arisen, including possibly the most contentious of all: were the fossil 'carpoids' really chordates? Sprinkle thinks this unlikely and that these highly unusual echinoderms represent convergence upon the 'flat-fish' design of asymmetrical adaptation to benthic living, presenting echinoderm workers with a remarkable group within an already unusual phylum, whose puzzles and challenges are well reviewed in this book.

David Nichols is Professor of Biological Sciences at the University of Exeter.

\section{Mathematics for physics}

\section{Michael Atiyah}

Topology and Geometry for Physicists. By Charles Nash and Siddhartha Sen. Academic Press: 1983. Pp. 310. £25, $\$ 39.50$.

GEOMETRY and physics have a long and entangled history. Euclidean geometry, with the help of Descartes, provided in due course the framework for the Newtonian Universe. Later, in the nineteenth century, the discovery of non-Euclidean geometries liberated mathematicians and led to a much wider range of geometrical thinking. One aspect of this was Riemann's differential geometry which eventually provided the mathematical foundations of Einstein's General Theory of Gravitation. A different but related strand was the development of the geometry of 'fibre bundles' by Elie Cartan and his followers. Together with the associated growth of topology (which might be described as 'qualitative geometry') this has been one of the most vigorous and successful branches of twentieth-century mathematics.

Quite remarkably all this modern geometry and topology, although apparently very abstract, has suddenly come into prominence, as it provides the mathematical setting for the latest and most promising theory of elementary particle physics - the so-called gauge theories. As a result physicists have found it necessary to come to terms with modern geometrical ideas, a task made more difficult by the different technical jargon employed by mathematicians and physicists.

Mathematical books are notoriously difficult to read. The requirements of formal proof lead to a ponderous and intimidating style, and there are few books that could be recommended to the enquiring physicist. It is to remedy this situation that Nash and Sen have written their book. It is intended for the physicist who would like to learn the rudiments of modern geometry and topology. As a result there is much more in the way of motivation and illustrative examples than is customary. Moreover the final chapters deal specifically with physical applications in both solid state and gauge theories.

Although the style is less formal than in standard treatments, the coverage is quite extensive and thorough, and the book could usefully be read by mathematics students. Altogether the authors have achieved their purpose and provided a work which should prove extremely useful in bridging the gap between geometry and physics.

Sir Michael Atiyah is at the Mathematical Institute, University of Oxford. 